\title{
Composición Corporal y el Efecto de un Programa de Fuerza Auxiliar para Prevenir Lesiones en Musculos Cuádriceps Femoral, Isquiotibiales y Bíceps Femoral en Jóvenes Universitarios Futbolistas
}

\author{
Body Composition and the Effect of an Auxiliary Force Program to Prevent Injuries in \\ Femoral quadriceps, Hamstrings and Biceps Femoris Muscles in Young College Players
}

\author{
Espinoza-Navarro, O.* \& Valle, S. ${ }^{* *}$
}

ESPINOZA-NAVARRO, O. \& VALLE, S. Composición corporal y el efecto de un programa de fuerza auxiliar para prevenir lesiones en músculos cuádriceps femoral, isquiotibiales y bíceps femoral en jóvenes universitarios futbolistas. Int. J. Morphol., 32(3):1095-1100, 2014.

RESUMEN: El objetivo de esta investigación fue conocer los efectos de un programa de fuerza auxiliar en la prevención de lesiones de los músculos: cuádriceps femoral, isquiotibiales y bíceps femoral en futbolistas universitarios de la ciudad de Madrid, independiente del programa normal de entrenamiento del club universitario. Veinticuatro jugadores universitarios de entre 19 a 28 años participaron en el estudio, separados en grupo control $(n=12)$ y grupo experimental $(n=12)$. En ambos grupos se evaluó inicialmente el desplazamiento y la velocidad (potencia), mediante un transductor de movimiento o "encoder". Posteriormente al grupo experimental se le aplicó un programa de fuerza auxiliar, para reforzar los músculos del muslo, por un período de 6 meses. El Comité de Ética/Bioética de la Universidad de Tarapacá, aprobó los protocolos de esta investigación. Los resultados muestran que el grupo experimental presenta un aumento significativo en la ganancia de fuerza/potencia, en relación al grupo control, expresada en watts $(1187,6 \pm 110,4$ vs. 1011,6 $\pm 110,4$, respectivamente). El porcentaje de recuperación de la fuerza fue significativamente mayor en el grupo experimental, con un $16,8 \%$ y $0,17 \%$ en el grupo de control. El porcentaje de jugadores lesionados en el grupo experimental fue significativamente menor que en el grupo control (16,6\% y 50\%, respectivamente). Se concluye que los programas preventivos de fuerza auxiliar, en los jugadores de futbol de entre 19 a 28 años, son eficaces en la prevención de lesiones musculares por la ganancia considerable de fuerza/potencia, aportando un importante beneficio económico y deportivo. Por lo tanto, se recomienda incluir estos programas de fuerza auxiliar en todos los clubes deportivos profesionales.

PALABRAS CLAVE: Lesiones; Fútbol; Fuerza muscular; España.

\section{INTRODUCCIÓN}

El fútbol es un deporte de intensidad elevada, donde una pierna soporta grandes cargas en movimiento y existe un contacto estrecho entre sus participantes. Aunque suele decirse que las lesiones deportivas son parte del fútbol, existe una toma de conciencia creciente en los profesionales para instaurar medidas preventivas y terapéuticas que disminuyan su incidencia, porque se está transformado en una verdadera epidemia, a nivel mundial, sobre todo las lesiones de la musculatura del muslo (Stolen et al., 2005).

A partir del Primer Congreso Mundial de Prevención de lesiones deportivas realizado en Oslo el 2005, quedó pa- tente la necesidad de establecer medidas preventivas basadas en la evidencia con el objetivo de disminuir las incidencias de algunas lesiones, especialmente las musculares y ligamentosas (Adamuz \& Nerin, 2006).

La composición corporal de los sujetos pocas veces es considerada en la selección de los jóvenes deportistas, poniendo más atención a las habilidades de los individuos, sin considerar que la masa muscular y la fuerza, son factores preponderantes en su rendimiento. Mayor estatura y masa muscular confieren a los deportistas la capacidad de correr más km por partido, saltar más alto, facilitar el rendimiento intermitente de

Profesor Titular, Laboratorio Biología de la Reproducción y Desarrollo, Departamento de Biología, Facultad de Ciencias, Universidad de Tarapacá, Arica, Chile.

** Kinesiólogo, Magíster en Ciencias de la Actividad Física, Universidad de Tarapacá, Arica, Chile. 
alta intensidad, característico de deportes altamente competitivos como el fútbol (Jorquera Aguilera et al., 2012).

Es la formación temprana del futbolista, la base del deportista, es ahí donde se centra el problema, ya que la mayoría desconoce los beneficios que otorga el entrenamiento de fuerza. Son frecuentes las lesiones en la etapa adulta, provocadas por los desbalances musculares, principalmente del tren inferior, refiriéndose al desequilibrio entre los músculos cuádriceps e isquiotibiales y musculatura del tronco (Reilly, 2003; Stolen et al.).

Trabajos de rendimiento físico han determinado la importancia del estudio epidemiológico en la prevención y tratamiento de las lesiones. Esto debido a las enormes diferencias musculares entre las diferentes actividades físicas. El éxito del deportista por tanto dependerá del tipo de musculatura y sus capacidades funcionales, sin olvidar la genética y las respuestas específicas, lo que condicionará su eficiencia mecánica (Panasiuk, 2009; Rodríguez-Matoso et al., 2012).

Los músculos cuádriceps y los llamados músculos isquiotibiales son los que presentan mayor predisposición a lesionarse con 31,7 y un 17\% de incidencia respectivamente. Según Osorio et al. (2009) informan que en los músculos "isquiotibiales", conformados por los músculos semitendinoso, semimembranoso y bíceps femoral, el más propenso a lesionarse durante las temporadas de juego, es el bíceps femoral, con un 53\% del total de lesiones en este grupo muscular. Es importante destacar que por cada lesión se considera una licencia de reposo aproximadamente de 18 días, generando pérdidas económicas muy altas a los clubes o asociaciones (Osorio et al.; Ortín, 2010). Kucera et al. (2005), determinan que los futbolistas tienen 2,6\% más posibilidad de lesionarse si previamente ha tenido una lesión. Esta situación es extrema si se analiza el deporte de aficionado donde cerca de un 50\% de los participantes sufre alguna lesión que le impide la práctica regular y continuada (Palmi, 2001).

Estas lesiones son cada día más comunes y se están transformando en una epidemia. Dentro de los factores de riesgo se destaca el desbalance muscular, fatiga, técnicas de entrenamiento deficiente, mala postura lumbar, etc. Los músculos del muslo son biarticulares muy potentes y con características anatómicas, fisiológicas y biomecánicas diferentes. Conocer el origen de las lesiones musculares, su epidemiología, su repercusión en el futbolista y los factores de riesgo que intervienen, se convierten en un campo de investigación importantísimo para todo profesional vinculado a la actividad física (Panasiuk). La prevención de lesiones implica aplicación de programas de entrenamiento, debidamente integrado en la planificación de la temporada de competencia, que está destinado a fortalecer las zonas musculares más susceptibles de lesionarse. En la práctica deportiva del fútbol esta zona está determinada por la musculatura del tren inferior (Naclerio \& Goss-Sampson, 2013).

Esta investigación presenta un fundamento científico, sobre los beneficios de la aplicación de un trabajo de fuerza auxiliar complementaria en estudiantes universitarios de la Universidad Europea de Madrid que practican fútbol, con la finalidad de prevenir lesiones de los músculos cuádriceps femoral, músculo semitendinoso, músculo semimembranoso y músculo bíceps femoral, muy comunes en la práctica de este deporte y que van de la mano con otras lesiones tales como rotura de ligamentos cruzados o meniscos.

\section{MATERIAL Y MÉTODO}

Este trabajo es de tipo experimental pre test - post test con un grupo control. Veinticuatro futbolistas de 19 a 28 años, de sexo masculino, de la liga nacional universitaria de fútbol de Madrid, integrantes del equipo de la Universidad Europea de Madrid, se separaron en grupo control ( $\mathrm{n}=12$ ) y grupo experimental $(n=12)$. Cada una de estas agrupaciones, fueron divididas en forma aleatoria. Se registraron los datos personales y se confeccionaron las fichas de variación de fuerza muscular pre-test y post-test, medido con un Transductor de movimiento o "encoder rotatorio" (Real Power Globus, Italia), conectado a una máquina de Multipower. El encoder es un sistema de cables giratorios que permite determinar con gran precisión, posición, tiempo y desplazamiento para calcular los valores de fuerza/potencia, a través de un software. Este dispositivo permite al cable desplazarse verticalmente, respetando una dirección ascendente durante la fase concéntrica y descendente durante la fase excéntrica de los movimientos.

Programa de fuerza auxiliar. Se realizó un trabajo, con una frecuencia de 2 veces por semana, 1 hora de duración, por un período de 6 meses. Cada sesión se inició con un calentamiento previo. La primera parte del programa consistió en un periodo de adaptación al entrenamiento de fuerza base, posteriormente integración de la musculatura del miembro inferior y musculatura de tronco, para mejorar la estabilidad articular, el tiempo de respuesta neuromuscular y la fuerza/potencia. Se trabajó con pesos submáximos, es decir $2-3$ series de 8 a 12 repeticiones al 55-85\%, trabajando la vertiente de fuerza potencia (Resistencia muscular: RM). La forma de recuperar entre series, fue mediante estiramientos activos (Behm \& Anderson, 2006; Jiménez et al., 2006; Gamble, 2007). 
Posteriormente se estableció la distribución de la muestra, a través de la técnica de muestreo aleatorio simple y mediante randomización se dividió la muestra en grupo control y grupo experimental. A continuación se recopila-

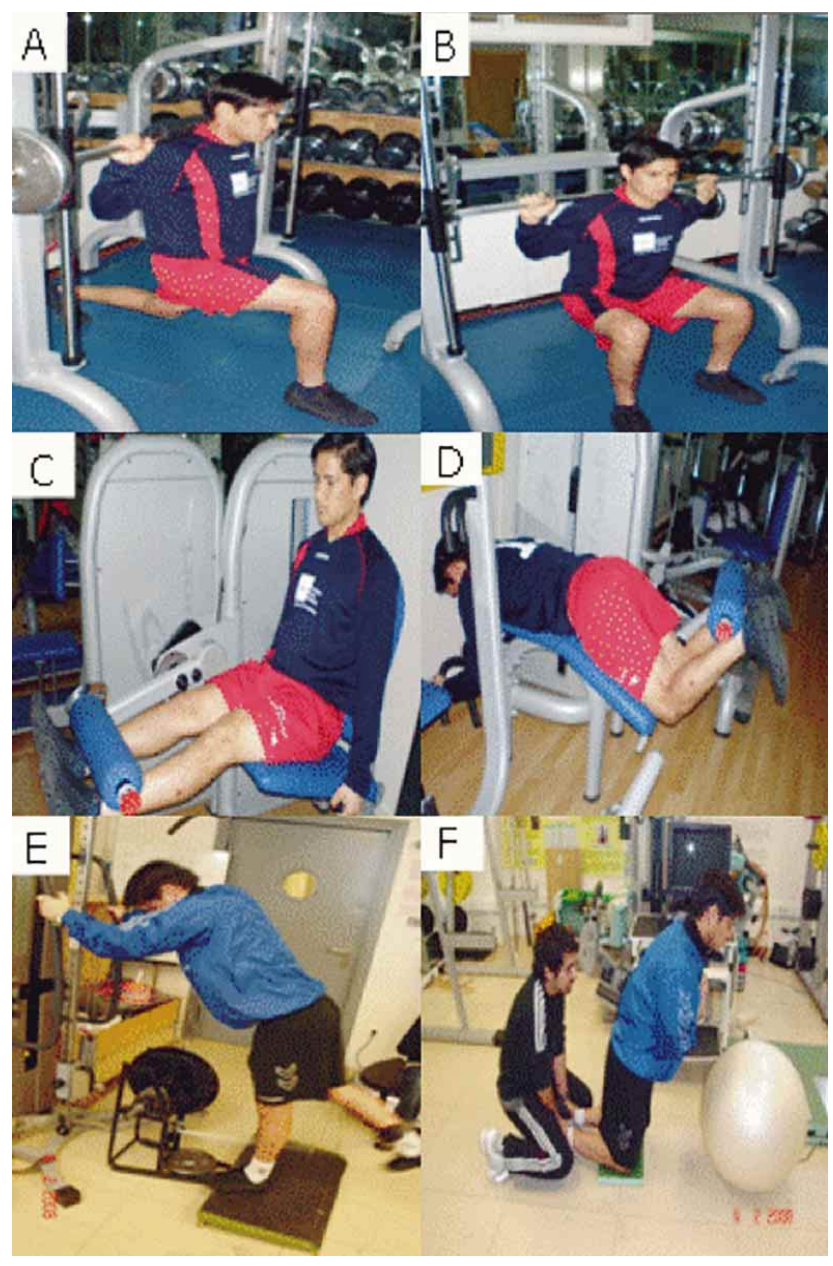

Fig. 1. Programa preventivo de fuerza auxiliar para los músculos cuádriceps femoral, isquiotibiales y músculo bíceps femoral. Expresión de los ejercicios para entrenamiento Fuerza/Potencia. A: Split frontal; B: Sentadilla en máquina multipower; C: Ejercicio de banco para músculos cuádriceps femoral; D Camilla para músculos isqiotibiales y músculo bíceps femoral; E: Patada trasera en paella para músculos cuádriceps femoral, isquiotibiales y músculo bíceps femoral; F: Ejercicio para músculo cuádriceps femoral "caída rusa". ron los datos obtenidos a partir de la evaluación de la Fuerza/Potencia Media (PM), de miembros inferiores, inicial y final de cada grupo. Se obtuvieron la medición Fuerza/Potencia Media inicial-final y el porcentaje de mejoría de ambos grupos. Los ejercicios aplicados en el programa de fuerza auxiliar para músculos del tren inferior se observan en la Figura 1.

Posteriormente se comparó la diferencia entre las mediciones finales de la variable dependiente (Fuerza de la musculatura de muslo); entre ambos grupos de estudio, cuantificada a través de medición de fuerza de miembros inferiores en un multipower conectado a un Encoder Rotatorio basándose en los datos de la potencia media. Finalmente, se comparó la diferencia observada entre el grupo control y experimental, de la evaluación post-test, con respecto al porcentaje de lesiones.

A partir de los datos obtenidos en la evaluación de Fuerza/PM, se calculó el promedio y la desviación estándar (DS), de los valores registrados, en ambos grupos de trabajo.

Análisis estadístico. Una vez obtenidos los resultados se trabajó con los estadísticos de promedios, desviación estándar, error estándar, y correlaciones. La significancia estadística de las diferencias dentro y entre grupos se realizó mediante las pruebas de t student y ANDEVA, considerando un $\mathrm{p}<0,05$.

\section{RESULTADOS}

En la Tabla I se muestran las mediciones de composición corporal de los grupos de estudios. Se observa que los valores de registros son muy similares en ambos grupos, control y experimental.

La Tabla II registra los resultados de la ganancia de Fuerza/ Potencia Media medida en watts, al inicio y al final de la aplicación del programa de fuerza auxiliar y los porcentajes de recuperación, para los músculos cuádriceps

Tabla I. Valores de la composición corporal de futbolistas jóvenes españoles, al inicio del estudio. Grupo experimental y control.

\begin{tabular}{llcccc}
\hline \multirow{2}{*}{ Grupo } & & Edad & Peso & Talla & IMC \\
\cline { 3 - 5 } & & $\mathbf{( a n ̃ o )}$ & $\mathbf{( k g )}$ & $(\mathbf{m})$ & $\mathbf{( k g / m 2})$ \\
\hline \multirow{2}{*}{$\begin{array}{l}\text { Control } \\
(\mathbf{n}=\mathbf{1 2})\end{array}$} & Promedio & 22,5 & 72,8 & 174,6 & 23,8 \\
& \pm DE & $\pm 3,5$ & $\pm 4,7$ & $\pm 4,8$ & $\pm 0,8$ \\
Experimental & (Min-Max) & $(20-28)$ & $(66-81)$ & $(169-182)$ & $(22,4-24,8)$ \\
$(\mathbf{n = 1 2})$ & Promedio & 22,66 & 73,6 & 175,3 & 24,1 \\
& \pm DE & $\pm 2,3$ & $\pm 6,9$ & $\pm 5,1$ & $\pm 1,1$ \\
& (Min-Max) & $(19-26)$ & $(64-83)$ & $(169-183)$ & $(22,1-25,3)$ \\
\hline
\end{tabular}

ŁDE: Desviación estándar. 
Tabla II. Ganancia de Fuerza/Potencia Media, al inicio y al final de la aplicación del programa de fuerza auxiliar para los músculos cuádriceps femoral, isquitibiales y músculo bíceps femoral (estudio de pre y post test). Porcentaje de recuperación, grupo control y experimental.

\begin{tabular}{|c|c|c|c|c|c|}
\hline \multicolumn{2}{|l|}{ Grupo } & $\begin{array}{c}\text { RMI } \\
(\mathbf{k})\end{array}$ & $\begin{array}{l}\text { F/PMI Pre test } \\
\text { (watts) }\end{array}$ & $\begin{array}{c}\text { F/PMF Post test } \\
\text { (watts) }\end{array}$ & $\begin{array}{l}\text { \% Recuperación } \\
\text { de la fuerza }\end{array}$ \\
\hline $\begin{array}{l}\text { Control } \\
(n=12)\end{array}$ & $\begin{array}{l}\text { Promedio } \\
\pm \text { DE } \\
\text { (Min-Max) }\end{array}$ & $\begin{array}{c}161,3 \\
\pm 17,5 \\
(138-188)\end{array}$ & $\begin{array}{c}1009,6 \\
\pm 92,2 \\
(904-1145)\end{array}$ & $\begin{array}{c}1011,6 \\
\pm 110,4 \\
(910-1150)\end{array}$ & 0,17 \\
\hline $\begin{array}{l}\text { Experimental } \\
(\mathbf{n = 1 2})\end{array}$ & $\begin{array}{l}\text { Prome dio } \\
\pm \text { DE } \\
\text { (Min-Max) }\end{array}$ & $\begin{array}{c}168,9 \\
\pm 18,7 \\
(140-197)\end{array}$ & $\begin{array}{c}1016,9 \\
\pm 69,8 \\
(915-110)\end{array}$ & $\begin{array}{c}1187,6^{*} \\
\pm 110,4 \\
(1002-1340)\end{array}$ & $16,8^{*}$ \\
\hline
\end{tabular}

RMI= Resistencia Muscular Inicial; F/PMI= Fuerza/Potencia Media Inicial; F/PMF=Fuerza/Potencia Media Final; \pm DS= Desviación estándar, *p<0,05.

Tabla III. Porcentaje de jugadores lesionados y no lesionados en musculatura de tren inferior, durante el periodo de competición en grupo control y experimental con un programa de fuerza auxiliar.

\begin{tabular}{lccccc}
\hline \multirow{2}{*}{ Grupo } & $\mathbf{n}$ & $\begin{array}{c}\text { Jugadores } \\
\text { lesionados }\end{array}$ & Sin lesiones & Con lesiones & Total \\
\cline { 3 - 6 } & & 6 & $\mathbf{\%}$ & $\mathbf{\%}$ & $\boldsymbol{\%}$ \\
\hline Control & 12 & 2 & 80 & 50 & 100 \\
Experimental & 12 & 2 & 70 & 16,6 & 100 \\
Total & 24 & 8 & 30 & 100 \\
\hline
\end{tabular}

femoral, músculo semitendinoso, músculo semimembranoso y músculo bíceps femoral (estudio de pre y post test). El grupo experimental registra un valor post test con un aumento significativo de ganancia Fuerza/Potencia de $1187,6 \pm 110,4$ watts, con un aumento significativo en el porcentaje de recuperación de un 16,8\%. El grupo control registra valores de $1011,6 \pm 110,4$ watts y un porcentaje de recuperación de $0,17 \%(* \mathrm{p}<0,05)$.

En la Tabla III se muestra el porcentaje de jugadores lesionados con y sin programa de fuerza auxiliar. El grupo experimental presenta una baja significativa en el porcentaje de lesiones con un 16,6\% de jugadores lesionados, respecto al grupo control sin programa de fuerza auxiliar con un $50 \%$ de jugadores lesionados $(* \mathrm{p}<0,05)$.

\section{DISCUSIÓN}

Según Hagglund et al. (2005), el fútbol se caracteriza por la intermitencia de sus acciones, donde de forma asistemática se intercalan esfuerzos de alta, moderada y baja intensidad, existiendo una permanente disputa con el adversario en donde el contacto físico es habitual y el riesgo de lesiones se incrementa con la intensidad del juego. Durante un partido de fútbol, se realizan distintas actividades con diferentes intensidades desde caminar o trotar ligeramente hasta realizar sprints o aceleraciones máximas (Stolen et al.). Si bien la carrera en diferentes intensidades es la acción más realizada, durante el partido esta se alterna con otro tipo de acciones de alta exigencia e intensidad como saltos, entradas, forcejeos para mantener la posición del jugador o del balón, frenos cambios de direcciones, controles, golpes y lanzamiento del balón, que aunque se realizan con un volumen inferior su importancia es decisiva para alcanzar el éxito (Hoff, 2006).

En la práctica del fútbol, los músculos más comprometidos son los músculos cuádriceps femoral, isquiotibiales y el músculo bíceps femoral. El cuádriceps está conformado por los músculos: vasto medial, vasto intermedio, el vasto lateral y el recto femoral. Su propósito es extender la rodilla y ayudar a estirar la pierna. Los músculos isquiotibiales, compuestos por los músculos: semitendinoso y semimembranoso y el bíceps femoral tienen por función acelerar el uso de las piernas al caminar o al correr (Garret \& Kirkendall, 2000).

Toda la gama de actividades e intensidad en el juego hacen proclive a los jugadores a gran cantidad de lesiones en estos grupos musculares. Naclerio \& Goss-Sampson proponen ejercicios de estiramiento activo específico, ejercicios de estiramiento, o ejercicios específicos del deporte, los cuales deberían integrarse a los programas regulares de entrenamiento.

Los resultados obtenidos en este estudio, luego de la aplicación de este programa de fuerza auxiliar, independiente del programa normal de entrenamiento durante 
la temporada de juegos, muestra un aumento en la fuerza y la potencia de los jugadores en relación a los jugadores sin programa auxiliar. Similares resultados encuentra Subramanium (2002), quien resalta la importancia de la fuerza muscular para las acciones específicas del juego.

Un factor importante en el rendimiento físico es la composición corporal. Nuestro estudio con jóvenes jugadores españoles, muestra somatotipo muy similares a otros estudios con jóvenes españoles y sudamericanos (Rivera, 2006; Zúñiga \& de León, 2007; Panasiuk; Herrero de Lucas, 2004; Jorquera Aguilera et al.). Esta homogeneidad interpoblacional en los patrones morfológicos resulta ser característico de este tipo de jugadores.

Referente a las lesiones en futbolistas Subramaniun, acota que "el fútbol es todo músculo: fuerza, resistencia, potencia, velocidad, coordinación, destreza, agilidad y flexibilidad. En un individuo desentrenado, la gran exigencia física durante el partido es la causa de la mayoría de las lesiones". Naclerio \& Goss-Sampson, recomiendan que con el fin de prevenir las "lesiones de los isquiotibiales", los programas deberían ser diseñados de manera que incluyan tanto la actividad excéntrica, como ejercicios de co-con- tracción estabilizadora de la rodilla. Además es importante que los entrenadores consideren la importancia de las correcciones, buscando una técnica deportiva específica y un buen control motor.

\section{CONCLUSIÓN}

La aplicación del programa preventivo de fuerza auxiliar, aplicado en estos jugadores universitarios de futbol de entre 19 a 28 años, fue eficaz enla prevención de lesiones musculares por la ganancia considerable de la Fuerza/Potencia. De ello se deriva un importante beneficio económico y deportivo. Por lo tanto, se recomienda su inclusión en los programas de entrenamiento en todos los clubes deportivos.

\section{AGRADECIMIENTOS}

Este trabajo fue subvencionado por el proyecto UTA Mayor No 4712-13, de la Universidad de Tarapacá, AricaChile

ESPINOZA-NAVARRO, O. \& VALLE, S. Body composition and the effect of an auxiliary force program to prevent injuries in femoral quadriceps, hamstrings and biceps femoris muscles in young college players. Int. J. Morphol., 32(3):1095-1100, 2014.

SUMMARY: The objective of this research was to determine the effects of a program of auxiliary force in the prevention of injuries to muscles: quadriceps femoris, hamstrings and biceps femoris in college football players of the city of Madrid, independent of the normal training program of university club. Twenty-four college players aged 19-28 years participated in the study, divided into experimental group $(n=12)$ and control group $(n=12)$. In both groups, the displacement and the velocity (potency) were initially assessed through a motion transducer or "encoder". Then in experimental group was implemented a program of auxiliary force for 6 months. The Ethics/Bioethics Committee at the Universidad of Tarapacá, approved the protocols of this research. The results shown in the experimental group, with program of auxiliary forces a significant increase in gain strength/potency, relative to the control group, expressed in watts (1187.6 \pm 110.4 vs. $1011.6 \pm 110.4$ respectively), the percentage recovery of force was $16.8 \%$ in the experimental group and 0.1 larger than bifurcation aneurysms until they

$7 \%$ in the control group. The percentage of injured players in the experimental group was significantly lower than the control group ( $16.6 \%$ and $50 \%$ respectively). We conclude that preventive programs auxiliary force in football players 19 and 28 years are effective in preventing muscle injuries by the considerable gain strength/potency, providing great economic and sporting benefits. Therefore it is recommended include these programs of auxiliary force in all sports clubs.

\section{KEY WORDS: Muscular Injuries; Football; Quadriceps; Femoral Biceps; Spain.}

\section{REFERENCIAS BIBLIOGRAFÍA}

Adamuz, F. \& Nerin, M. El fisioterapeuta en la prevención de lesiones del deporte. Rev. Fisioter. (Guadalupe), 5(2):31-6, 2006.

Behm, D. G. \& Anderson, K. G. The role of instability with resistance training. J. Strength Cond. Res., 20(3):716-22, 2006.

Gamble, P. An integrated approach to training core stability. Strength Condit. J., 29(1):58-68, 2007.
Garrett, W. \& Kirkendall, D. Exercise and Sports Sciences. Philadelphia, Lippincott, Williams and Wilkins, 2000.

Hagglund, M.; Waldén, M.; Bahr, R. \& Ekstrand, J. Methods for epidemiological study of injury to professional football players: developing the UEFA model. Br. J. Sports Med., 39(6):340-6, 2005. 
Herrero de Lucas, A. Cineantropometría: Composición corporal y somatotipo de futbolistas que desarrollan su actividad física en equipos de la Comunidad Autónoma de Madrid. Tesis Doctoral, Facultad de Medicina, Departamento de Anatomía y Embriología, Universidad Complutense de Madrid, España, 2004.

Hoff, J. Training and testing physical capacities for elite soccer player. J. Sports Sci., 23(6):573-82, 2006.

Jiménez, D. J. F. Lesiones musculares en el deporte. Int. J. Sport Sci., 3(2):55-67, 2006

Jorquera Aguilera, C.; Rodríguez Rodríguez, F.; Torrealba Vieira, M. I. \& Barraza Gómez, F. Body Composition and Somatotype of Chilean Soccer Players Sub 16 y Sub 17. Int. J. Morphol., 30(1):247-52, 2012.

Kucera, K. L.; Marshall, S. W.; Kirkendall, D. T.; Marchak, P. M. \& Garrett, W. E. Jr. Injury history as a risk factor for incident injury in youth soccers. Br. J. Sports Med., 39(7):462-72, 2005.

Naclerio, F. \& Goss-Sampson, M. The effectiveness of different exercises protocols to prevent the incidence of hamstring injury in athletes. OA Sports Med., 1(2):11, 2013.

Ortín, F. Factores psicológicos y socio-deportivos y lesiones en jugadores de fútbol semiprofesional y profesional. Tesis Doctoral. Universidad de Murcia, España, 2010.

Osorio, C.; Rossi, R.; Hidalgo, R. \& Lizana, M. Relación entre flexibilidad y fuerza muscular en isquiotibiales y su incidencia en lesiones musculares en jóvenes futbolistas. Rev. Kinesiol., 28:139, 2009.

Palmi, G. J. Visión psico-social en la intervención de la lesión deportiva. Cuad. Psicol. Deporte, 1(1): 69-79, 2001.

Panasiuk, A. Estudio retrospectivo sobre la prevalencia de las principales lesiones de los futbolistas profesionales en el Uruguay, Abril 1997-Mayo 2007. Rev. AKD, 41:8-10, 2009.

Reilly, T. Motion analysis and physiological demands in soccer. In: Reilly, T. \& Williams, M. (Ed.). Science and soccer. 2nd ed. London, Routledge, 2003. pp.59-72.

Rivera, J. Valoración del somatotipo y proporcionalidad de futbolistas universitarios mexicanos respecto a futbolistas profesionales. Rev. Int. Med. Cienc. Act. Fis. Deporte, 6(21):16-28, 2006.

Rodríguez-Matoso, D.; García-Manso, J. M.; Sarmiento, S.; de Saa, Y.; Vaamonde, D.; Rodríguez-Ruiz, D. \& da Silva-Grigoletto, M. E. Evaluación de la respuesta muscular como herramienta de control en el campo de la actividad física, la salud y el deporte. Rev. Andal. Med. Deporte, 5(1):28-40, 2012.

Stolen, T.; Chamari, K.; Castagna, C. \& Wisloff, U. Physiology of Soccer. Sports Med., 35(6):501-36, 2005.
Subramanium, S. Effects of combined strength and kick coordination training on soccer kick biomechanics in amateur players. Scand. J. Med. Sci. Sports, 16(2):102-10, 2002.

Zúñiga, U. \& de León, L. Somatotype of semiprofessional soccer players classified by their position in the game. Int. J. Sport Sci., 3(9):29-36, 2007.

\author{
Dirección para Correspondencia: \\ Omar Espinoza-Navarro \\ Profesor Titular \\ Laboratorio Biología de la Reproducción y Desarrollo \\ Departamento de Biología, Facultad de Ciencias \\ Universidad de Tarapacá \\ Arica \\ CHILE
}

Email: oespinoz@uta.cl

Recibido: 09-07-2014

Aceptado: 22-08-2014 